\title{
The RXTE/ASM X-ray light curve of GX301-2
}

\author{
D. A. Leahy ${ }^{\star}$ \\ Department of Physics, University of Calgary, Calgary, Canada T2N 1N4 \\ Received 11 April 2002 / Accepted 17 May 2002

\begin{abstract}
GX301-2 is an accreting neutron star in an eccentric orbit $(e=0.462)$ with a massive early-type stellar companion. GX301-2 accretes from the stellar wind and exhibits regular X-ray flares approximately 1.4 days prior to periastron passage. Long-term continuous X-ray monitoring of GX301-2 by the RXTE/All-Sky-Monitor has now been carried out for a period of 5 years. These data now comprise the best observations of the orbital X-ray light curve. The main flux peak just prior to periastron is clearly seen, as well as a secondary broad peak near apastron. CGRO/BATSE observations have provided new improved orbital parameters. The constraint on the orbital inclination is examined. Then the GX301-2 orbital light curve is modeled using wind, disk and stream models. The conclusion is that only a stellar wind plus mass stream is consistent with the data. The wind model is further supported by calculations of the stream flow from the primary and the expected orbital phases that the neutron star crosses the stream.
\end{abstract}

Key words. stars: neutron - stars: individual: GX301-2 - stars: emission line, Be - X-rays: stars

\section{Introduction}

GX 301-2 (also known as 4U 1223-62) is a pulsar with a $680 \mathrm{~s}$ rotation period, in a 41.5 day eccentric orbit (Sato et al. 1986). The mass function is $31.8 M_{\odot}$, making the minimum companion mass $35 M_{\odot}$ for a $1.4 M_{\odot}$ neutron star. The companion Wray 977 has a B2 Iae spectral classification (Parkes et al. 1980).

The neutron star flares regularly in X-rays approximately 1-2 days before periastron passage, and several stellar wind accretion models have been proposed to explain the magnitude of the flares and their orbital phase dependence (e.g. Koh et al. 1997; Leahy 1991; Haberl 1991). The modeling by Leahy (1991) and Haberl (1991) was done using TENMA and EXOSAT observations, respectively, which cover many short data sets spaced irregularly over orbital phase. More recently better orbital phase coverage has been obtained by CGRO/BATSE (Koh et al. 1997), which however has much lower sensitivity than the previous studies.

The X-ray spectrum of GX301-2 has been studied by TENMA (Leahy \& Matsuoka 1990; Leahy et al. 1989a; Leahy et al. 1989b) and ASCA measurements (Saraswat et al. 1996). The latter study illustrates the complexity of the GX301-2 spectrum. Four components are necessary: i) an absorbed power law with high column density; ii) a scattered power law with much lower column density; iii) a thermal component with temperature of $0.8 \mathrm{keV}$; iv) a set of six emission lines (including the iron line at $6.4 \mathrm{keV}$ ). Of the above, ii) and iv) are due to reprocessing in the stellar wind of Wray 977.

\footnotetext{
^ e-mail: leahy@iras.ucalgary.ca
}

Long-term monitoring of GX301-2 has now been carried out by the All-Sky-Monitor (ASM) on board RXTE. This provides the best measurement of the orbital light curve of GX301-2 yet. Here the results of the analysis of the RXTE/ASM data on GX301-2 including modeling of the orbital light curve are presented.

\section{RXTE/ASM data analysis}

The ASM on RXTE (Levine et al. 1996) consists of three scanning shadow cameras (SSC's), each with a field of view of $6^{\circ}$ by $90^{\circ} \mathrm{FWHM}$. The SSC's are rotated in a sequence of "dwells" with an exposure typically of $90 \mathrm{~s}$, so that most of the sky can be covered in one day. The dwell data are also averaged for each day to yield a daily-average. The RXTE/ASM dwell data and daily-average data were obtained from the ASM web site. The data reduction to obtain the count rates and errors from the satellite observations was carried out by ASM/RXTE team, and the procedures are described at the web site. The ASM count rates used here include the full energy range band as well as the three sub-bands $1.3-3.0 \mathrm{keV}, 3.0-5.0 \mathrm{keV}$ and 5.0-12.1 keV. The data covered the time period MJD 50087.2 to MJD 52284.5. Figure 1 shows the light curve for the whole time period using the daily-average full energy band data. The regular outbursts every 41.5 day orbital cycle are seen, as well as the variability from cycle to cycle.

The orbital parameters of GX301-2, updated with the BATSE observations (Koh et al. 1997) and used for the current study are as follows. $P_{\text {orb }}=41.498$ days, $a_{x} \sin (i)=368.31 \mathrm{lt}-\mathrm{s}$, eccentricity $e=0.462$, longitude of periastron $\omega=310^{\circ}$, time of periastron passage $T_{0}=$ MJD 48802.79. 


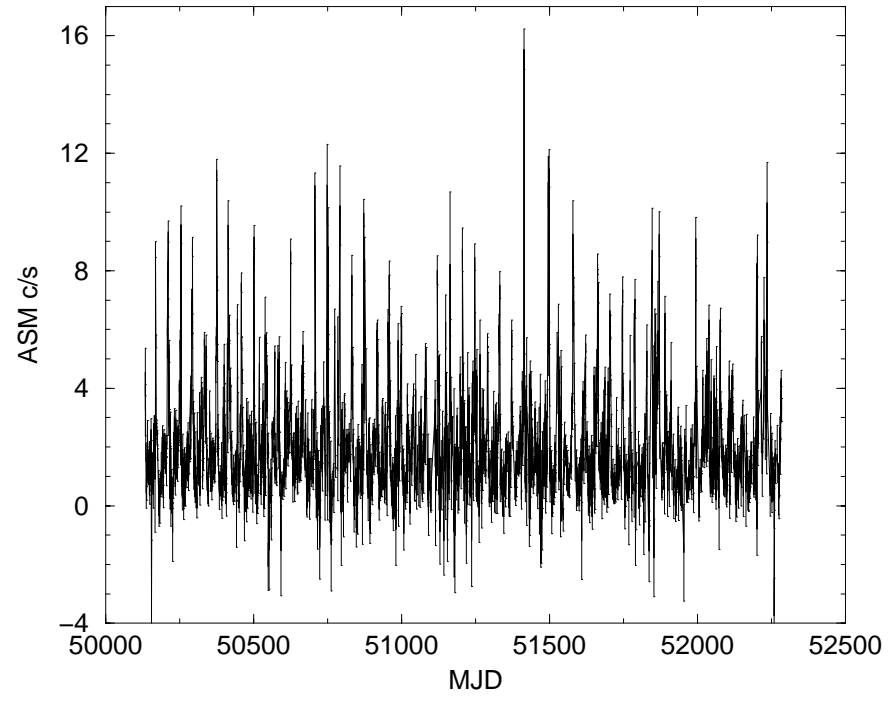

Fig. 1. GX301-2 ASM full band light curve using daily-average data.

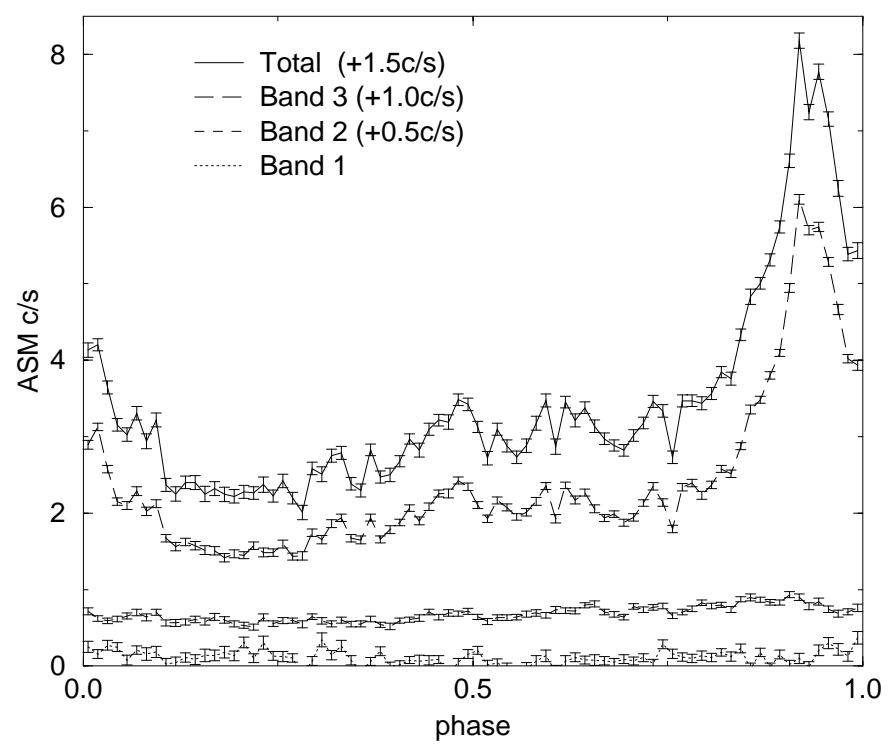

Fig. 2. Orbital light curves of GX301-2 in the three RXTE/ASM energy bands and total energy band from the ASM dwell data folded at 41.498 days.

An epoch folding analysis was carried out on the full energy range band data, with the $\chi^{2}$ statistic used to assess the reality of variability at any given trial period (e.g. see Leahy et al. 1983 for a description of the epoch folding method). The daily-average and dwell data were folded into 80 phase bins for periods in a range around 41.5 days, giving maximum $\chi^{2}$ values of 6900 and 17022 , respectively, at a period of 41.498 days. This is identical to the known orbital period.

The dwell data gives a more significant light curve. This is probably due to the 1 day averaging in the daily data which results in smoothing the main flux peak in the folded light curve. The dwell data is used for this reason in preference to the dailyaverage data in the analysis that follows. The three RXTE/ASM sub-bands were epoch folded. The orbital light curve for these bands and the full energy range band are shown in Fig. 2 with orbital phase zero defined by the time of periastron passage, $T_{0}$.

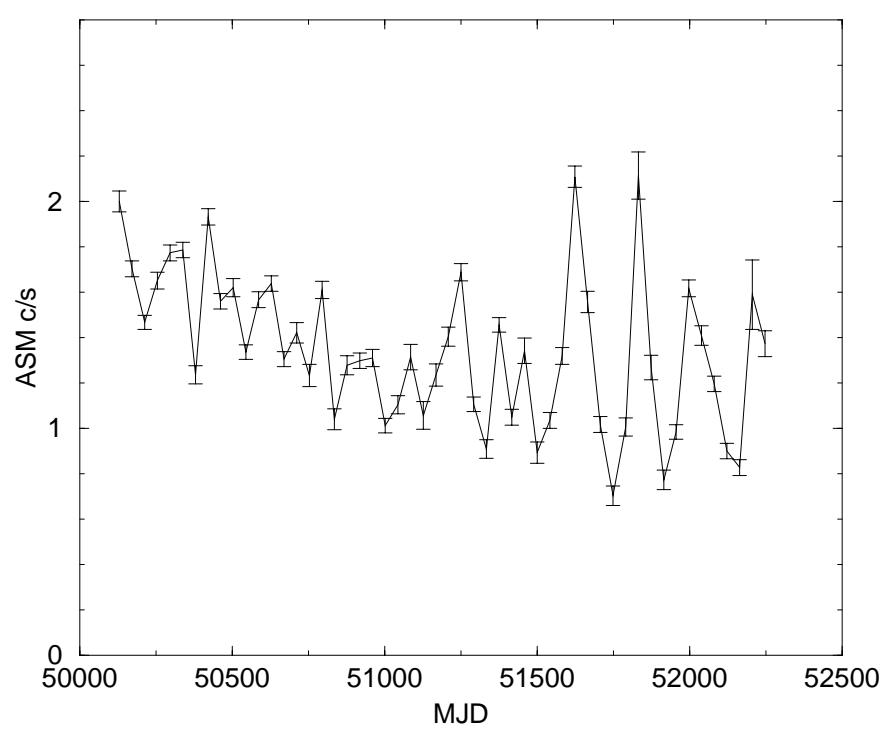

Fig. 3. Long-term light curve of GX301-2 with timebins of 41.498 days, for total energy band ASM dwell data.

GX301-2 shows a significant variability above statistical uncertainties in intensity from orbit to orbit. This is illustrated in Fig. 3 which shows the RXTE/ASM data over the entire observation period with timebins equal to one orbital period. The rms variability is $0.33 \mathrm{ASM} \mathrm{c} / \mathrm{s}$ compared to a mean error of $0.044 \mathrm{ASM} \mathrm{c} / \mathrm{s}$; the variability is real at greater than 7 sigma significance. The earlier dates show lower variability than the later dates and there is also a secular decrease in the mean flux in the amount of $-0.0002 \mathrm{ASM} \mathrm{c} / \mathrm{s} /$ day. However the length of the data set is not long enough to establish a long term trend, and the flux is consistent with no secular decrease after $\sim$ MJD 51200. The data were tested for long term periodicities by examining: a) $\chi^{2}$ vs. period for epoch folding for periods up to 500 days; and b) the power spectrum at frequencies less than $1 / P_{\text {orb }}$. No periodicities were detected. The power spectrum is flat to low frequencies, consistent with white noise (uncorrelated fluctuations) and showing no evidence for red noise. Figure 4 shows the orbital light curves for the total time period and 4 different time intervals. It is seen that the variability seen in Fig. 3 is primarily due to variability in the outburst peak near orbital phase 0.9 . The folded light curves are consistent with eachother between orbital phases 0.1 to 0.8 .

\section{Constraint on the orbital inclination}

The orbital inclination of GX301-2 is not constrained by the pulse timing data alone, but by including constraints on the mass and radius of the companion star, Wray 977. This is discussed by Koh et al. (1997), and an updated discussion is given here. The exact form of the Roche potential for a synchronously rotating star is computed here, rather than the standard simple approximation. The surface area of the critical Roche surface (or mean radius defined by the surface area) is the correct quantity to compare to the radius derived from the luminosity, rather than other measures of the Roche lobe such as the Roche radius. 


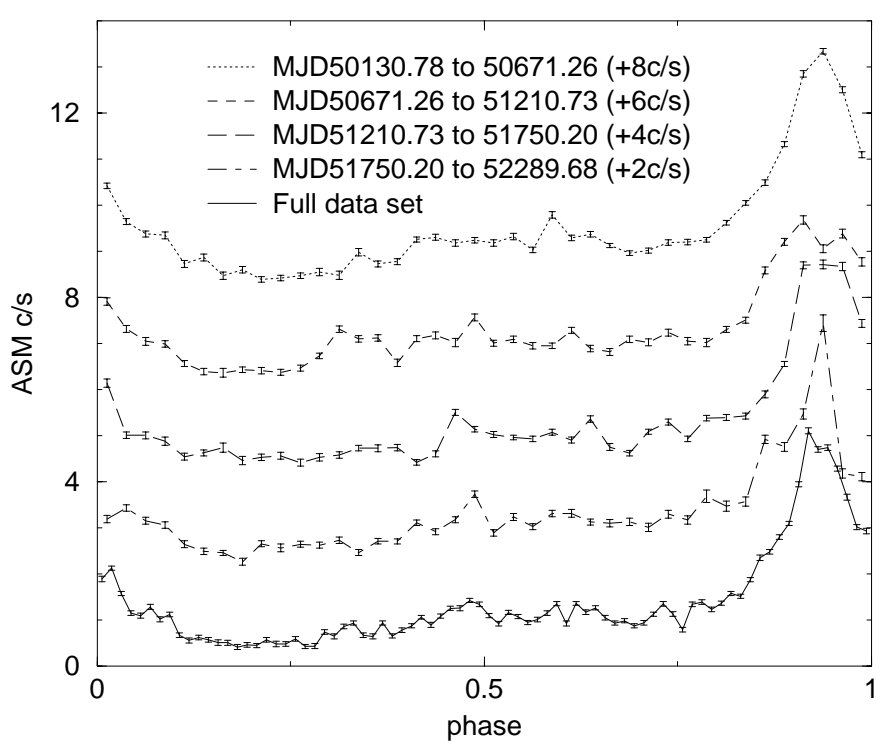

Fig. 4. Orbital light curves of GX301-2 for 4 different time intervals compared with that for the total time period. The total energy band ASM dwell data was folded at 41.498 days.

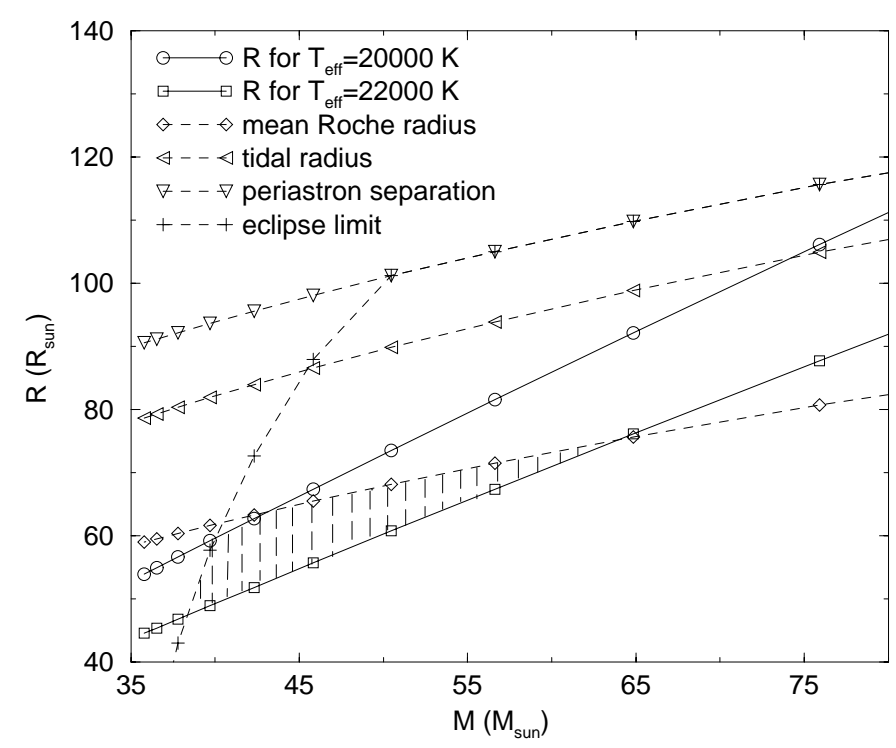

Fig. 5. Mass-radius constraints for GX301-2 (see text): the massradius relation for lower and upper values of $T_{\text {eff }}$; the mean Roche radius; the tidal radius at periastron; the periastron separation; and the maximum radius allowed by absence of eclipse. The allowed region for a star inside the Roche critical surface is shown by the hashed region.

The companion (primary) mass, $M_{\mathrm{c}}$, depends on the system inclination. The mass, radius and angular velocity all affect the modelling of the stellar wind, which is needed to model the X-ray light curve. The orbital parameters given in the previous section give the value of the mass function $f(M)=$ $\left(M_{\mathrm{c}} \sin (i)\right)^{3} /\left(M_{x}+M_{\mathrm{c}}\right)^{2}$. Here the neutron star (secondary) mass is assumed to be $M_{x}=1.4 M_{\odot}$. If a different value were taken it would only have a small effect on the derived primary mass as a function of inclination. The possible masses span a large range: from about $35 M_{\odot}$ to more than $80 M_{\odot}$ (as inclination goes to zero, the mass goes to $\infty$ ).

The stellar radius is constrained by optical observations. The observed flux and effective temperature give the radius if the distance is known. However, it is not well known. The bolometric flux corrected for reddening is $F_{\text {bol }} \simeq 1.45 \times$ $10^{-6} \mathrm{erg} \mathrm{cm}^{-2} \mathrm{~s}^{-1}$ and effective temperature is $T_{\text {eff }}=$ 20 000-22 $000 \mathrm{~K}$ (Koh et al. 1997). The spectral type is B1 Ia+ (Kaper et al. 1995). The mass-luminosity relation of Koh et al. (1997), derived from the stellar models of Shaller et al. (1992), is adopted here. This gives the mass-radius relations plotted in Fig. 5 for $T_{\text {eff }}=20000 \mathrm{~K}$ and $T_{\text {eff }}=22000 \mathrm{~K}$.

Another constraint is provided by the absence of any eclipse of the neutron star throughout the entire orbit. A numerical calculation of minimum radius to cause an eclipse was carried out, with the results shown in Fig. 5 by the dashed line with plus signs. This constraint gives lower limits on mass (upper limits on inclination, calculated using $f(M)$ ) which are $38.4 M_{\odot}\left(76.8^{\circ}\right)$ for $T_{\text {eff }}=22000 \mathrm{~K}$ and $40.0 M_{\odot}\left(73.6^{\circ}\right)$ for $T_{\text {eff }}=20000 \mathrm{~K}$.

The radius of Wray 977 is constrained so that the star remains stable against gravitational forces from the companion. The constraint is a function of the geometry of the orbit, which is a function of inclination, and thus of mass from $f(M)$. The simplest constraint is that the radius must be less than the periastron separation, which is plotted in Fig. 5 (top curve). The upper limits on mass (lower limits on inclination) are $122 M_{\odot}$ $\left(41^{\circ}\right)$ for $T_{\mathrm{eff}}=22000 \mathrm{~K}$ and $88 M_{\odot}\left(47^{\circ}\right)$ for $T_{\mathrm{eff}}=20000 \mathrm{~K}$. A stronger constraint is that the star must be inside its tidal radius at periastron. The tidal radius as a function of mass is plotted in Fig. 5 by dashed line with left-pointing triangles. The tidal radius constraint gives upper limits on mass (lower limits on inclination) of $106.3 M_{\odot}\left(43.2^{\circ}\right)$ for $T_{\text {eff }}=22000 \mathrm{~K}$ and $74.2 M_{\odot}\left(50.6^{\circ}\right)$ for $T_{\text {eff }}=20000 \mathrm{~K}$.

A stronger yet constraint is that the star must be within its critical Roche lobe. The Roche potential is given by:

$\Omega=-\frac{G M_{1}}{r_{1}}-\frac{G M_{2}}{r_{2}}-\frac{\omega^{2} \rho^{2}}{2}$

$r_{1}$ is the distance to the center of the primary star, $r_{2}$ is the distance to the center of the secondary star $\rho$ is the distance to the axis of rotation of the binary, and $\omega$ is the angular velocity of rotation of the system. The origin of coordinates can be taken at the primary star with the $z$-axis normal to the binary orbital plane, and $x$ axis from the primary to the secondary. From this one can find the value of the critical potential $\Omega_{\mathrm{c}}$, where the potential has its saddle point along the line joining the two stars by writing the potential as a function of $x, y, z$ and setting the partial derivative of $\Omega$ to zero along the $\mathrm{x}$ axis $(y=z=0)$. Then one can find the two dimensional surface for which $\Omega(x, y, z)=\Omega_{\mathrm{c}}$, otherwise known as the critical Roche lobe. The mean Roche radius has been calculated, which is defined as the spherical radius which gives the same surface area as the critical Roche lobe. It is plotted in Fig. 5 by the dashed line with diamonds, and gives upper limits on mass and lower limits on inclination which are: $64.0 M_{\odot}$ and $54.3^{\circ}$ for $T_{\text {eff }}=22000 \mathrm{~K}$; and $43.1 M_{\odot}$ and $43.2^{\circ}$ for $T_{\text {eff }}=20000 \mathrm{~K}$. 
The allowed region in the mass radius plane is shown in Fig. 5 by the hashed region.

For the modeling below, calculations were done with inclinations between $55^{\circ}$ and $76^{\circ}$. The case with $T_{\text {eff }}=21000 \mathrm{~K}$ and inclination of $62^{\circ}$ (called here the standard case) is near the center of the allowed region. It has mass of $50.5 M_{\odot}$ and stellar radius of $66.7 R_{\odot}$. For this case, the observed optical flux from Wray 977 yields a distance of $4.1 \mathrm{kpc}$.

\section{Comparing models to the data}

A comparison of various models to the RXTE/ASM orbital light curve is made by means of $\chi^{2}$ minimization using the nonlinear conjugate gradient method. GX301-2 has a significant absorption by its stellar wind in soft X-rays (e.g. Leahy 1991). This shows up well in Fig. 2 above: Band 1 and Band 2 data are affected significantly by absorption but Band $3(5-12.1 \mathrm{keV})$ is almost completely free of absorption effects. This is expected as the photoelectric cross-section is very small above $3-4 \mathrm{keV}$. This is also confirmed by the consistency in shape of the Band 3 light curve with the BATSE light curve (Koh et al. 1997), although the RXTE/ASM light curve here is of significantly better statistical quality. Thus the $5-12.1 \mathrm{keV}$ band flux is taken as a measure of the X-ray luminosity of the pulsar.

\subsection{Wind model}

A spherically symmetric wind has been shown to not produce the main flux peak prior to periastron (Leahy 1991; Haberl 1991). However, neither of those studies included an azimuthal component in the wind. This component is small but does shift the phase of the main flux peak. The wind velocity law here is taken to be of form (e.g. Castor et al. 1975): $v_{\mathrm{w}}(r)=$ $v_{\mathrm{o}}\left(1-R_{\mathrm{s}} / r\right)^{\beta}+c_{\mathrm{s}}$ with $\beta=1$ and $c_{\mathrm{s}}$ the speed of sound in the wind. There is an additional azimuthal component to the velocity. This is due to stellar rotation and is equal to the equatorial rotation velocity of the primary at $r=R_{\mathrm{s}}$, with $R_{\mathrm{S}}$ the radius of the primary. The azimuthal component decreases with $r$ due to angular momentum conservation. The accretion rate is taken to be the Bondi-Hoyle accretion rate (e.g. Leahy 1991). The wind model was fit to the RXTE/ASM data with free parameters $v_{\mathrm{o}}$ and normalization, using the standard case for the inclination, masses, stellar radius and effective temperature. The normalization can be related to the mass-loss rate after conversion of ASM counts/s to flux, conversion to luminosity given a distance to GX301-2, and applying assumed accretion efficiency and neutron star mass. The wind parameter $\beta$ has a small effect on the fits, and was fixed at 1.0 after comparing results with different $\beta$. The best-fit wind model is shown in Fig. 6 and has $v_{\mathrm{o}}=186 \mathrm{~km} \mathrm{~s}^{-1}$. A peak just prior to periastron does occur but not early enough, nor does the shape of the light curve fit the data. The best-fit $\chi^{2}$ is $1.01 \times 10^{4}$ for 78 degrees of freedom (d.o.f.).

\subsection{Wind plus disk model}

An additional dense, slowly expanding circumstellar disk around Wray 977 was postulated by Pravdo et al. (1995) to

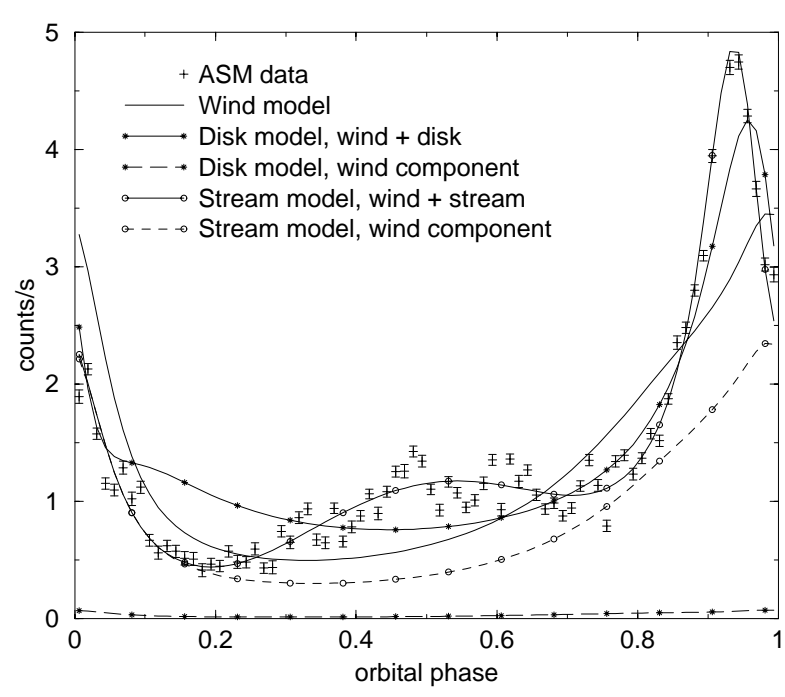

Fig. 6. Folded light curve of GX301-2 compared to the models (see text).

explain the CGRO/BATSE light curve, which shows the preperiastron main flux peak and also a secondary flux peak at pre-apastron. The RXTE/ASM data shows that the apastron fluxpeak is very broad (see Fig. 2 here) and is centered approximately on orbital phase 0.55 (consistent with the CGRO/BATSE phase). A circumstellar disk is crossed by the pulsar twice per orbital period at positions $180^{\circ}$ apart. This translates to a phase difference which is quite different than 0.5 due to the eccentric orbit. To test whether a disk could explain the two peaks seen in the RXTE/ASM light curve, three different types of disk models were fitted to the data. All three models included the above stellar wind model as a component. The first model included a constant thickness, constant density disk. The disk had a Gaussian density profile perpendicular to the disk plane and had arbitrary orientation except that the disk plane passed through the primary star. The disk tilt parameter with respect to the binary plane is nearly degenerate with the disk thickness parameter. Thus the disk tilt was fixed at $45^{\circ}$ although several other values of tilt were used also, without improving the fit results. The least squares fitting gave a best-fit disk density of zero, leaving only the wind component with the same best-fit parameters as for the wind model above. The second disk model included a constant thickness, $1 / r$ density disk. The least squares fitting for this also gave a best-fit disk density of zero. The third model included a constant angular thickness disk with radial density profile the same as that of the stellar wind. The resulting best-fit of this third disk model is shown in Fig. 6, with the wind component shown separately. The bestfit $\chi^{2}$ is $5.00 \times 10^{3}$ for 75 degrees of freedom (d.o.f.). The fit is unacceptable. The reason is disk models in general are unacceptable is that they cannot fit both the pre-periastron and apastron flux peaks with the known orbital constraints from the pulse timing.

\subsection{Wind plus stream model}

This type of model was used by Haberl (1991) and Leahy (1991) in different forms to fit the less complete data from 
Table 1. Stream model best-fit parameters vs. inclination.

\begin{tabular}{lrrrrrr}
\hline \hline$I$ & \multicolumn{2}{c}{$55^{\circ}$} & \multicolumn{2}{c}{$62^{\circ}$} & \multicolumn{2}{c}{$76^{\circ}$} \\
\hline$v_{0}\left(\mathrm{~km} \mathrm{~s}^{-1}\right)$ & $195 \pm 10$ & $200 \pm 10$ & 211 & \pm 11 \\
$\phi_{1}$ & $-67^{\circ} \pm 1^{\circ}$ & $-67^{\circ} \pm 1^{\circ}$ & $-65^{\circ} \pm 1^{\circ}$ \\
$\sigma_{1}$ & $25^{\circ} \pm .3^{\circ}$ & $25^{\circ} \pm .3^{\circ}$ & $25^{\circ} \pm .3^{\circ}$ \\
$\alpha_{1}$ & $1.59 \pm .04$ & $1.47 \pm .03$ & $1.19 \pm .03$ \\
$\phi_{2}$ & $172^{\circ} \pm 1^{\circ}$ & $173^{\circ} \pm 1^{\circ}$ & $175^{\circ} \pm 1^{\circ}$ \\
$\sigma_{2}$ & $21^{\circ} \pm 1^{\circ}$ & $21^{\circ} \pm 1^{\circ}$ & $21^{\circ} \pm 1^{\circ}$ \\
$\alpha_{2}$ & $1.95 \pm .12$ & $2.25 \pm .13$ & $1.99 \pm .13$ \\
\hline
\end{tabular}

EXOSAT and TENMA. The basic idea, as proposed by Stevens (1988), is that a gas stream flows out from the point on the primary facing the neutron star. Here two separate analyses were done. One is fitting a phenomenological stream model to the RXTE/ASM data. The other is a numerical calculation of the spiral stream geometry as a function of binary phase. The fitting is described first, the calculated geometry described next, and the consistency between the two described last.

The stream is taken to have a geometry such that the stream is crossed twice by the neutron per binary orbit. This is motivated by the observations of two flux peaks at orbital phases 0.93 and 0.55 , and confirmed by the stream calculations in the next section. For the luminosity, only the stream properties where the neutron star crosses the stream are important. The model parameters are the stellar wind velocity $v_{0}$, the position angle $\phi_{1}$, over-density $\alpha_{1}$ and Gaussian width $\sigma_{1}$ of the stream for the first crossing, and $\phi_{2}, \alpha_{2}$ and $\sigma_{2}$ for the second crossing. The best fit stream model is shown in Fig. 6 assuming the standard case (inclination $62^{\circ}$, mass $50.5 M_{\odot}$, $\left.T_{\text {eff }}=21000 \mathrm{~K}\right)$. It has a best-fit $\chi^{2}$ of 901 for 72 degrees of freedom (d.o.f.). The main contribution to $\chi^{2}$ is from the large scatter in the data compared to the smooth model curve between binary phases 0.2 and 0.8 . Taking this into account, the model does produce a satisfactory explanation of the light curve. The best fit parameters for inclinations, $I$, of $55^{\circ}, 62^{\circ}$ and $76^{\circ}$, are given in Table 1.

\subsection{Dynamical stream calculation}

Here the location of a gas stream from the primary star is calculated. The stream's origin is the point on the primary along the line of centers. The stream is taken to have the same radial velocity as the stellar wind, given above. The azimuthal velocity is determined by conservation of angular momentum. The stellar angular velocity, $\omega$ is described by the parameter $f: \omega=$ $f \times \omega_{\text {orb }}+(1-f) \times \omega_{\text {per }}$, with $\omega_{\text {orb }}$ is the average orbital angular velocity $\left(2 \pi / P_{\text {orb }}\right)$ and $\omega_{\text {per }}=\omega_{\text {orb }}(1+e)^{0.5} /(1-e)^{1.5}=3.06 \omega_{\text {orb }}$ is the periastron angular velocity. Thus the primary is taken to be rotating at some angular velocity between $\omega_{\text {orb }}$ and $\omega_{\text {per }}$. The large difference in $\omega_{\text {orb }}$ and $\omega_{\text {per }}$ is due to the high eccentricity of the orbit. For any given choice of orbital phase, the stream position can be integrated using the radial and azimuthal velocities. The stream takes an Archimedes-type spiral pattern, with a shape which varies with orbital phase. The shape change is due to the variation in angular velocity of the point of origin

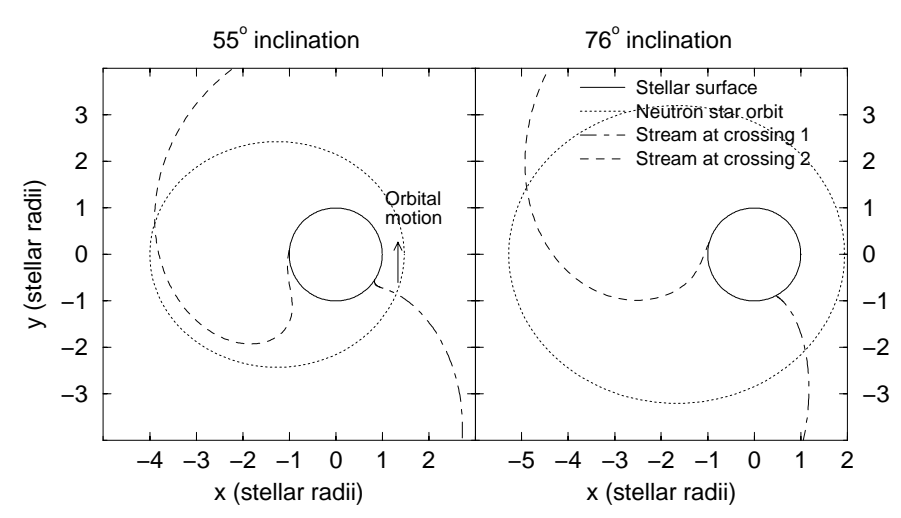

Fig. 7. Instantaneous locations of the stream for two different orbital phases. Left panel: $55^{\circ}$ inclination: stream position at orbital phase .969 , orbital angle $327^{\circ}$ (crossing 1) and at orbital phase .444 , orbital angle $172^{\circ}$ (crossing 2). Right panel: $76^{\circ}$ inclination: stream position at orbital phase .936 , orbital angle $298^{\circ}$ (stream crossing 1) and orbital phase .399 , orbital angle $165^{\circ}$ (stream crossing 2).

of the stream on the primary, which is caused by the variable angular velocity of the neutron star.

To illustrate the stream Fig. 7 (left panel) shows the stream at two different orbital phases for the case of inclination $55^{\circ}$ and rotation parameter $f=0.4$. The stream, at the distance of the neutron star, has an angular velocity which varies with orbital phase due to the changing stream shape: higher angular velocity near apastron and lower angular velocity near periastron. Due to the eccentric orbit, the neutron star also has a changing angular velocity: lower angular velocity near apastron and higher angular velocity near periastron. Thus the neutron star passes the stream near periastron (called stream crossing 1) and the stream passes the neutron star near apastron (called stream crossing 2). The two particular orbital phases shown in Fig. 7 are just those where the stream crossings occur. To illustrate the effect of inclination on the stream shapes and stream crossings (and on the system size and orbit) Fig. 7 (right panel) shows the case inclination $76^{\circ}$ and rotation parameter $f=0.4$. The change in stream shape with orbital phase is smaller as the inclination increases, and the stream crossings move to earlier orbital phases.

The rotation rate of the primary star affects the stream angular velocity and position vs. orbital phase. Thus it affects the orbital phases where the neutron star crosses the stream. This is illustrated in Fig. 8 for orbital inclinations of $55^{\circ}$ and $76^{\circ}$. The solid lines give the orbital angles of the two stream crossings as a function of $f$. The dotted lines give the stream crossings derived from the light curve fits, which vary slowly with inclination. As one increases the inclination from $55^{\circ}$ to $76^{\circ}$, the agreement between calculated and the fit for the first crossing (near $5.1 \mathrm{rad}$ ) occurs for $f$ decreasing from 0.66 to 0.42 . The agreement between calculated and the fit for the second crossing (near $3 \mathrm{rad}$ ) occurs for $f$ increasing from 0.43 to 0.59 . Both crossings match for $f \simeq 0.51$ at inclination of $\simeq 65^{\circ}$.

\section{Conclusions}

The RXTE/ASM light curve is the best measurement yet of the orbital light curve of GX301-2. It shows the main 


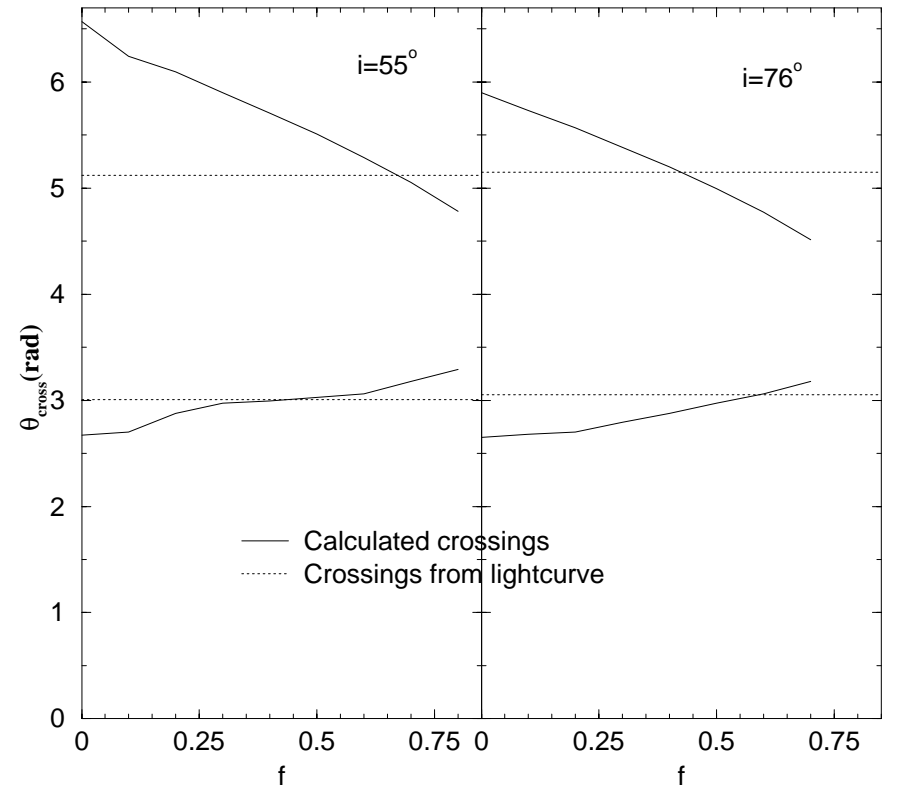

Fig. 8. Orbital angles of the two calculated stream crossings vs. $f$, the stellar rotation parameter (see text). Left panel, $55^{\circ}$ inclination: solid lines-; dotted line: the two stream crossings from the light curve fit. Right panel: $76^{\circ}$ inclination: solid lines- orbital angles of the two calculated stream crossings vs. $f$, the stellar rotation parameter; dotted line: the two stream crossings from the light curve fit.

pre-periastron peak and also a broad apastron flux peak. An examination of the time variability shows that the variability is primarily in the main flux peak and not in the rest of the light curve (Fig. 4). In terms of the wind plus stream model described above, this means the variability is primarily due to variability in the stream and not in the wind.

The constraints on the inclination, mass and radius of GX301-2 were examined more accurately than done previously. The constraints were derived using the mass-luminosity relation derived from Shaller et al. (1992), so depend on $T_{\text {eff }}$, which observationally is between $20000 \mathrm{~K}$ and $22000 \mathrm{~K}$. For the orbit not to give eclipses of the neutron star, upper limits on inclination (lower limits on mass and radius) are: $76.8^{\circ}$ (38.4 $M_{\odot}$ and $\left.47.5 R_{\odot}\right)$ for $T_{\text {eff }}=22000 \mathrm{~K}$; and $73.6^{\circ}\left(40.0 M_{\odot}\right.$ and $59.6 R_{\odot}$ ) for $T_{\text {eff }}=20000 \mathrm{~K}$. For the companion to fit inside its Roche lobe, lower limits on inclination (upper limits on mass and radius) are: $54.3^{\circ}\left(64.0 M_{\odot}\right.$ and $\left.75.2 R_{\odot}\right)$ for $T_{\text {eff }}=22000 \mathrm{~K}$; and $43.2^{\circ}\left(43.1 M_{\odot}\right.$ and $\left.63.7 R_{\odot}\right)$ for $T_{\text {eff }}=20000 \mathrm{~K}$. The case with $T_{\text {eff }}=21000 \mathrm{~K}$ and inclination of $62^{\circ}$ is near the center of the allowed region, with mass of $50.5 M_{\odot}$ and stellar radius of $66.7 R_{\odot}$. This case has a distance of $4.1 \mathrm{kpc}$ in order to yield the observed optical flux from Wray 977.

The light curve analysis was carried out for wind, disk and stream models. The only model consistent with the data has a stellar wind component plus two stream crossings by the neutron star per orbit. The orbital angles of the crossings of the stream centers are well determined (to $\pm 1^{\circ}$ ).

A single stream (density enhancement) is expected to flow out from the primary star, with its origin at the point on the primary facing the neutron star, as described by Stevens (1988).
A calculation of the expected stream position was carried out here. The stream shape changes with binary phase (e.g. Fig. 7) due to the varying angular velocity of the origin point of the stream on the primary. This gives the stream a larger angular velocity near apastron and a smaller angular velocity near periastron. The neutron star however has a smaller angular velocity near apastron and larger angular velocity near periastron, resulting in the neutron star overtaking the stream near periastron and the stream overtaking the neutron star near apastron. Figure 7 illustrates the stream at the orbital positions of these two crossings, for two different system inclinations. The stream shape and the orbital angles of the crossings also depend on the parameter $f$ which characterizes the stellar angular rotation $\omega$ $\left(\omega=f \times \omega_{\text {orb }}+(1-f) \times \omega_{\text {per }}\right)$. For $f \simeq 0.51$ at inclination of $\simeq 65^{\circ}$, both of the calculated stream crossings agree with those derived from the light-curve fitting.

The stream model described here is fairly simple. Yet it is able to fit the light curve and to give agreement with the timing of both stream crossings. This is strong support that the stream model is the correct physical cause for the observed light curve. An estimate of the expected stream width can be made assuming the stream expands laterally at the speed of sound $\left(\simeq 20 \mathrm{~km} \mathrm{~s}^{-1}\right.$ at $\left.20000 \mathrm{~K}\right)$. The wind flow time, from the stream calculation, (e.g. for the case of inclination $55^{\circ}$ ) out to the first and second stream crossings are $0.15 P_{\text {orb }}$ and $0.38 P_{\text {orb }}$, yielding angular half-widths of the stream at these two crossings of $8.0^{\circ}$ and $9.2^{\circ}$, resp. These values do not change much with system inclination, and are within a factor of $\simeq 2$ of the values from the light curve fits.

In summary, the RXTE/ASM data analysis here has provided strong support for the Stevens (1988) model for a gas stream in the stellar wind of the massive X-ray binary GX301-2.

Acknowledgements. DAL acknowledges support from the Natural Sciences and Engineering Research Council.

\section{References}

Castor, J., Abbott, D., \& Klein, R. 1975, ApJ, 195, 157

Haberl, F. 1991, ApJ, 376, 245

Kaper, L., Lamers, H., Ruymaekers, E., van den Heuvel, E., \& Zuiderwijk, E. 1995, A\&A, 300, 446

Koh, D., Bildsten, L., Chakrabarty, D., et al. 1997, ApJ, 479, 933

Leahy, D. 1991, MNRAS, 250, 310

Leahy, D., \& Matsuoka, M. 1990, ApJ, 355, 627

Leahy, D. A., Matsuoka, M., Kawai, N., \& Makino, F. 1989, MNRAS, 236, 603

Leahy, D. A., Matsuoka, M., Kawai, N., \& Makino, F. 1989, MNRAS, 237, 269

Leahy, D. A., Elsner, R., \& Weisskopf, M. 1983, ApJ, 272, 256

Levine, A., Bradt, H., Cui, W., et al. 1996, ApJ, 469, L33

Parkes, G., Mason, K., Murdin, P., \& Culhane, J. 1980, MNRAS, 191, 547

Pravdo, S., Day, C., Angelini, L., et al. 1996, ApJ, 454, 872

Saraswat, P., Yoshida, A., Mihara, T., et al. 1996, ApJ, 463, 726

Sato, N., Nagase, F., Kawai, N., et al. 1986, ApJ, 304, 241

Shaller, G., Schaerer, D., \& Maeder, G. 1992, A\&AS, 96, 269

Stevens, I. R. 1988, MNRAS, 232, 199 